PROCEEDINGS OF THE

AMERICAN MATHEMATICAL SOCIETY

Volume 36. No. 2, December 1972

\title{
SELECTION OF REPRESENTING MEASURES FOR INNER PARTS
}

\author{
PETER D. TAYLOR
}

\begin{abstract}
AbSTRACr. If a compact convex set $K$ has an inner part $\Delta$ then there is a selection of pairwise boundedly absolutely continuous representing measures for $\Delta$ if and only if $K$ is finite dimensional.
\end{abstract}

Let $K$ denote a compact convex set in a LCTVS, $A(K)$ the affine continuous real functions on $K, \mathscr{P}(K)$ the set of regular Borel probability measures on $K$. Let $\Phi: \mathscr{P}(K) \rightarrow K$ be the map which associates to each measure $\mu$ its barycentre. Then $\Phi$ is affine, weak* continuous, and onto $K$. If $\Phi(\mu)=x$ we say $\mu$ represents $x$.

If $L$ is any convex set, $x, y \in L$ and $r>0$, we say $[x, y]$ extends by $r$ in $L$ if $x+r(x-y) \in L$ and $y+r(y-x) \in L$. We write $x \sim y$ if $\exists r>0$ such that $[x, y]$ extends by $r$ in $L$. This is an equivalence relation on $L$ and the equivalence classes are the parts of $L$. It is easy to show that $\Phi$ carries parts into parts: If $\Pi$ is a part of $\mathscr{P}(K)$ then $\Phi(\Pi)$ is contained in a part of $K$. Conversely if $\Delta$ is a part of $K$ and $F$ is any finite subset of $\Delta$ then there exists a part $\Pi$ of $\mathscr{P}(K)$ such that $F \subset \Phi(\Pi)$. Indeed if $F=\left\{x_{1}, x_{2}, \cdots, x_{n}\right\}$ choose $y_{i}$ and $z_{i}$ in $K$ such that $x_{1} \in\left(y_{i}, z_{i}\right)$, the open line segment with endpoints $y_{i}$ and $z_{i}$, and $x_{i} \in\left(y_{i}, x_{1}\right)(2 \leqq i \leqq n)$. If $\Phi\left(\mu_{i}\right)=y_{i}$ and $\Phi\left(v_{i}\right)=z_{i}$ for $\mu_{i}, v_{i} \in \mathscr{P}(K)$, then the part $\Pi$ containing $\sum\left(\mu_{i}+v_{i}\right) /(2 n-2)$ satisfies $F \subset \Phi(I I)$. Indeed since $x_{1} \in\left(y_{i}, z_{i}\right)$ for each $i$, we can clearly find a representing measure $\omega$ for $x_{1}$ in $\Pi$. Since $x_{i} \in\left(y_{i}, x_{1}\right)$, an affine combination of $\mu_{i}$ and $\omega$ yields a representing measure for $x_{i}$ in $\Pi$.

Thus if $\Delta$ is a part of $K$ one might ask whether

$$
\Delta=\Phi(\Pi) \text { for some part } \Pi \text { of } \mathscr{P}(K) .
$$

Indeed Bear posed this question in [3] and reproduced an example of Har'kova [4] to show that (1) need not hold if $\mathscr{P}(K)$ is replaced by $\mathscr{P}(\Gamma)$ where $\Gamma$ is the Shilov boundary of $A(K)$.

Since two probability measures $\mu$ and $\nu$ on $K$ are in the same part of $\mathscr{P}(K)$ if and only if $\mu \leqq k v$ and $\nu \leqq k \mu$ for some $k$, condition (1) asserts

Received by the editors September $11,1970$.

AMS (MOS) subject classifications (1970). Primary 52A20, 31 B10; Secondary 46E10, 28A40.

(c) American Mathematical Society 1973 
the existence for $\Delta$ of a selection of representing measures on $K$ which are pairwise boundedly absolutely continuous. There are two special cases when (1) is true for all parts $\Delta$ of $K$. One is when $K$ is a simplex, for then there are unique maximal representing measures $[6, \S 9]$, the other when $K$ is finite dimensional (Theorem 1 ).

Let $K^{i}=\{x \in K:(\forall y \in K)(\exists r>0) x+r(x-y) \in K\}$. It can happen that $K^{i}=\varnothing$, but if $K^{i} \neq \varnothing$ it is a part of $K$ called the inner part. Finite dimensional convex sets, for example, always have nonempty inner parts. In Theorem 1 we show that if $\Delta=K^{i} \neq \varnothing$ then (1) holds for $\Delta$ if and only if $K$ is finite dimensional.

First some preliminaries. If $L$ is a compact convex set, $x, y \in L$ and $x \sim y$; let

$$
d(x, y)=\inf \{\log (1+1 / r):[x, y] \text { extends by } r\} .
$$

In [3, Lemma 3.4] it is shown that $d$ is a metric on each part of $L$, called the part metric. Now denote by $d$ and $D$ the part metrics on $K$ and $\mathscr{P}(K)$ respectively and let

$$
b(x, r)=\{y \in K: d(x, y) \leqq r\} \quad \text { and } \quad B(\mu, r)=\{v \in \mathscr{P}(K): D(\mu, v) \leqq r\} .
$$

LEMMA 1. Suppose $\Delta$ is a part of $K, \Pi$ a part of $\mathscr{P}(K)$, and $\Delta=\Phi(\Pi)$. Then there exist $\mu \in \Pi$ and positive numbers $M$ and $k$ such that if $x=\Phi(\mu)$ then

$$
b(x, \log (1+1 / M)) \subset \Phi(B(\mu, \log k)) .
$$

Proof. If $v \in \Pi$ then the sets $\Phi(B(v, r))$ are closed in the part metric topology. Indeed suppose $x_{n}=\phi\left(\mu_{n}\right)$ with $\mu_{n} \in B(v, r)$ and $d\left(x_{n}, x\right) \rightarrow 0$. Choose a subset $\mu_{n_{\alpha}}$ converging weak* to $\mu$. Since $B(v, r)$ is weak* closed (easy to check), $\mu \in B(\nu, r)$. Since $\Phi$ is weak* continuous, $x_{n_{z}}$ converges in $K$ to $\Phi(\mu)$. But since $d\left(x_{n_{x}}, x\right) \rightarrow 0, x_{n_{\alpha}}$ converges in $K$ to $x$, hence $x=\Phi(\mu) \in \Phi(B(\nu, r))$. (It is an easily verified general fact that in any part of a compact convex set the part metric topology is stronger than the relativized compact topology.)

Since $\Delta=\Phi(\Pi)=\bigcup_{n=1}^{\infty} \Phi(B(v, n))$ and the part metric on $\Delta$ is complete $[1, \S 3]$, the Baire category theorem tells us that we can find $x \in \Delta$ and integers $h$ and $M$ such that $b(x, \log (1+1 / M)) \subset \Phi(B(\nu, h))$. Choose $\mu \in \Pi$ such that $\Phi(\mu)=x$ and choose $k$ such that $B(\nu, h) \subset B(\mu, \log k)$.

Lemma 2. Suppose $x \in K^{i}$. Then $\exists \delta>0$ such that

$$
y \in K \Rightarrow x+\delta(x-y) \in K .
$$

Proof. Let $H=K-x$. Then $0 \in H^{i}$ and so $H \cap-H$ is closed, convex and absorbs each point of $H$ and $-H$. Since $H$ is compact, convex, 
$H \cap-H$ absorbs $H$ [5, Corollary 10.2]. Thus $\exists \delta>0$ such that $\delta H \subset H \cap$ $-H \subset-H$. Thus

$$
y \in K \Rightarrow y-x \in H \Rightarrow \delta(y-x) \in-H \Rightarrow x+\delta(x-y) \in K .
$$

If $A$ is a normed linear space and $\varepsilon \geqq 0$ let $B_{\varepsilon}=\{h \in A:\|h\| \leqq \varepsilon\}$.

Lemma 3. Suppose $E$ is a normed linear space and $G$ is a weak* closed subspace of the dual space $E^{*}$. Suppose $x \in E^{*}, r \geqq 0$ and $\left(x+B_{r}\right) \cap G=\varnothing$. Then $\exists f \in E$ such that $\|f\|=1, f(G)=0$ and $f(x)>r$.

Proof. $x+B_{r}$ is weak* compact and $G$ is weak* closed. Hence $\exists f \in E$ such that $\|f\|=1, f(G)<\alpha$ and $f\left(x+B_{r}\right) \geqq \alpha$ for some $\alpha$. Since $G$ is a subspace, $\alpha>0$ and $f(G)=0$. Since $\|f\|=1$ we can find $y \in B_{r}$ such that $f(y)>r-\alpha$. Then $x-y \in x+B_{r}$ so $f(x-y) \geqq \alpha$ hence $f(x) \geqq \alpha+$ $f(y)>r$.

Now for the main theorem. We always think of $K$ as embedded in the Banach space $A(K)^{*}$ with the weak* topology. The norm of $A(K)^{*}$ provides a metric topology on $K$ which we will refer to as the norm topology.

THEOREM 1. Suppose $\Delta=K^{i} \neq \varnothing$. Then the following are equivalent.

(1) $\Delta=\Phi(\Pi)$ for some part $\Pi$ of $\mathscr{P}(K)$.

(2) $K$ is finite dimensional.

Proof. $\quad(1) \Rightarrow(2)$. Suppose (1) and suppose that $K$ is metrizable. We will show that, in this case, $K$ is finite dimensional. Then we will reduce the general case to this one.

We first show that $K$ is norm separable. If $\mu \in \Pi$ then $\Pi \subset L^{1}(\mu)$ (via Radon Nikodym), and the norm topology that $\Pi$ gets from $L^{1}(\mu)$ is the same as the norm topology it gets as a subset of $\mathscr{C}(K)^{*}$. Indeed if $g, h \in$ $L^{1}(\mu)$ then

$$
\begin{aligned}
\sup _{f \in \mathcal{C}\left(K^{\prime}\right):\|f\|_{\infty}=1} \int f(g-h) d \mu & =\|g d \mu-h d \mu\| \\
& =\sup _{f \in L^{\infty}:\|f\|_{\infty}=1} \int f(g-h) d \mu=\|g-h\|_{1},
\end{aligned}
$$

where $\|\cdot\|$ denotes the variation norm in the Banach space $\mathscr{M}(K)$ of Radon measures on $K$. Since $L^{1}(\mu)$ is separable ( $K$ is metrizable), $\Pi$ is norm separable in $\mathscr{C}(K)^{*}$. Since $\Phi$ is the restriction to $\mathscr{P}(K)$ of the natural, norm-decreasing surjection $\Phi: \mathscr{C}(K)^{*} \rightarrow A(K)^{*}, \Delta=\Phi(\Pi)$ is norm separable. Since $\Delta=K^{i}$ is norm dense in $K, K$ is norm separable.

Now we show that $K$ is norm compact. Since $K$ is norm complete it will be enough to find for any $\varepsilon>0$ a finite set $F \subset A(K)^{*}$ such that $K \subset F+B_{2 \varepsilon}$. So suppose $\varepsilon>0$. Choose $\mu, M, k$ and $x$ from Lemma 1 and $\delta$ from 
Lemma 2 so that $\delta(1+1 / M) \leqq 1$. Since $K$ is norm separable, we can cover $K$ with countably many balls of norm radius $r=\varepsilon \delta / 2 M$. A finite number of these balls contains all but at most $\gamma=\varepsilon \delta / 2 k M$ of the measure $\mu$. Let $P$ be a finite dimensional subspace of $A(K)^{*}$ containing $x$ and the centres of these finitely many balls.

We claim that $K \subset P+B_{\varepsilon}$. Indeed suppose $y \in K$ but $y \notin P+B_{\varepsilon}$. Let $z=x+(\delta / M)(y-x)$. Then $z \in K$ and $d(x, z) \leqq \log (1+1 / M)$. Indeed $x+M(x-z)=x+\delta(x-y)$ which is in $K$ by Lemma 2 , and $z+M(z-x)=$ $x+\delta(1+1 / M)(y-x)$ which is in $K$ since $\delta(1+1 / M) \leqq 1$. So by Lemma 1 we can choose $v \in B(\mu, \log k)$ such that $z=\Phi(v)$. An easy computation shows that $d v=g d \mu$ with $1 / k \leqq g \leqq k$. Also, since $P$ is weak* closed and $y \notin P+B_{\varepsilon}$ we can find $f \in A(K)$ such that $\|f\|=1, f(P)=0$ and $f(y)>\varepsilon$ (Lemma 3). Then

$$
\begin{gathered}
f(z)=(\delta / M) f(y)>\varepsilon \delta / M, \text { and } \\
v(f)=\int f g d \mu=\int_{|f| \leqq r} f g d \mu+\int_{|f|>r} f g d \mu \\
\leqq r \int g d \mu+\|f\| k \cdot \mu(\{|f|>r\}) \leqq r+k \gamma=\varepsilon \delta / M
\end{gathered}
$$

(where $\mu(\{|f|>r\}) \leqq \gamma$ since $|f(w)|>r \Rightarrow \mathfrak{w} \notin P+B_{r}$ ). Since $f \in A(K)$ and $\Phi(v)=z$ we must have $v(f)=f(z)$, a contradiction.

So $K \subset P+B_{\varepsilon}$. Hence $K \subset\left[\left(K+B_{\varepsilon}\right) \cap P\right]+B_{\varepsilon}$. Now $\left(K+B_{\varepsilon}\right) \cap P$ is finite dimensional and norm bounded, so relatively norm compact, and we can choose a finite set $F \subset A(K)^{*}$ so that $F+B_{\varepsilon}$ contains it. Hence $K \subset F+B_{2 \varepsilon}$.

So $K$ is norm compact. We deduce that the unit ball $B_{1}$ of $A(K)^{*}$ is norm compact. Indeed it follows from the Hahn Banach Theorem that every element of $A(K)^{*}$ is given by a Radon measure on $K$. Use the Hahn decomposition of this measure and the fact that any probability measure on $K$ has a barycentre in $K$ to deduce that, for any $\lambda \in B_{1}$, there exists $k, h \in K$ and $0 \leqq x, \beta \leqq 1$ such that

$$
\lambda(f)=\alpha f(k)-\beta f(h) \quad(f \in A(K)) .
$$

Thus $B_{1}$ is contained in a continuous image of $K \times K \times[0,1] \times[0,1]$, and is norm compact. It follows that $A(K)^{*}$ is finite dimensional, and so is $K$.

Now drop the metrizability assumption; suppose $K$ has (1) but is not finite dimensional. Choose a countably infinite, linearly independent sequence $\left\{f_{n}\right\} \subset A(K)$ such that $\left\|f_{n}\right\| \leqq 2^{-n}$. Define the map $\Psi$ from $K$ into $l^{2}$ by $\Psi(x)_{n}=f_{n}(x) . \Psi$ is affine and continuous, hence maps $K$ onto a compact convex subset $H$ of $l^{2}$. From Lemma 4 below $H^{i}=\Psi\left(K^{i}\right) \neq \varnothing$. Since every $x \in K^{i}$ has a representing measure in $\Pi$, every $h \in H^{i}$ has a 
representing measure in II $\circ \Psi^{-1}=\left\{\mu \circ \Psi^{-1}: \mu \in \mathrm{II}\right\}$. Since II is a part of $\mathscr{P}(K), \Pi \circ \Psi^{-1}$ is contained in a part of $\mathscr{P}(H)$ (from linearity of the map $\mu \rightarrow \mu \circ \Psi^{-1}$ ). So $H$ has property (1) and since it is metrizable it is, by the first part of the proof, finite dimensional. This contradicts the linear independence of $\left\{f_{n}\right\}$.

Lemma 4. Suppose $K$ and $H$ are convex sets and $K^{i} \neq \varnothing$. Suppose $\Psi: K \rightarrow H$ is affine and onto. Then $H^{i}=\Psi^{i}\left(K^{i}\right)$.

Proof. Clearly $\Psi^{i}\left(K^{i}\right) \subset H^{i}$. Assume $x \in H^{i}$. Choose $z^{\prime} \in K^{i}$ and let $z=\Psi\left(z^{\prime}\right)$. Since $x \in H^{i}, x=\lambda z+(1-\lambda) w$ for some $w \in H, 0<\lambda<1$. Choose $w^{\prime} \in K$ such that $\Psi\left(w^{\prime}\right)=w^{\prime}$. Then if $x^{\prime}=\lambda z^{\prime}+(1-\lambda) w^{\prime}$ we have $\Psi\left(x^{\prime}\right)=x$ and $x^{\prime} \in K^{i}$ since $z^{\prime} \in K^{i}$ and $0<\lambda<1$. So $x \in \Psi\left(K^{i}\right)$.

(2) $\Rightarrow(1)$. Suppose $K$ is of dimension $m$ and is in fact contained in $R^{m}$. If $x \in K^{i}$ then $K$ contains an open line segment containing $x$ in the direction of each coordinate axis. From the convexity of $K$ we deduce that $K$ and hence $K^{i}$ contains an open ball in $R^{m}$ containing $x$. Hence $K^{i}$ is open in $R^{m}$.

Choose $\left\{z_{i}\right\}$, a countable dense subset of $E(K)$. Let $\mu=\sum_{1}^{\infty}\left(\delta\left(z_{i}\right) / 2^{i}\right)$ $(\delta(z)=$ delta measure at $z)$. We will show $K^{i} \subset \Phi(\Pi)$ where $\Pi$ is the part of $\mathscr{P}(K)$ containing $\mu$. Choose $y \in K^{i}$. Let $\Phi(\mu)=x \in K$. Since $y \in K^{i}$ we can choose $w \in K^{i}$ and $1>\alpha>0$ so $y=\alpha . r+(1-\alpha) w$. Choose $\varepsilon>0$ so, $\forall g \in R^{m}$,

$$
\left\|g-w^{\|}\right\|<\varepsilon \Rightarrow g \in K \quad\left(\|\cdot\| \text { is Euclidean norm in } R^{m}\right) .
$$

Choose $n$ so $\left\{z_{1}, z_{2}, \cdots, z_{n}\right\}$ is an $\varepsilon$-net for $E(K)$. We claim that $\| \in$ $\operatorname{co}\left\{z_{1}, z_{2}, \cdots, z_{n}\right\}$. If not $\exists \gamma \in R^{m},\|\gamma\|=1$ such that $(\gamma, w)>\left(\gamma, z_{i}\right)$ for $1 \leqq i \leqq n$. Now $w+\varepsilon \gamma \in K$. Thus $\exists z \in E(K)$ so that

$$
(\gamma, z) \geqq(\gamma, w+\varepsilon \gamma)=(\gamma, w)+\varepsilon>\left(\gamma, z_{i}\right)+\varepsilon, \quad 1 \leqq i \leqq n .
$$

It follows that $\left\|z-z_{i}\right\|>\varepsilon$ if $1 \leqq i \leqq n$. This contradicts the choice of $n$.

So $\| \in \operatorname{co}\left\{z_{1}, z_{2}, \cdots, z_{n}\right\}$. This provides a measure $\nu \in \mathscr{P}(K)$ such that $\Phi(v)=w$ and $v \leqq 2^{n} \mu$. Clearly the probability measure $\alpha \mu+(1-\alpha) v$ represents $y$. It is in II since $\alpha>0$ and $\alpha \mu \leqq \alpha \mu+(1-\alpha) \nu \leqq\left(\alpha+2^{n}\right) \mu$.

Remarks. (1) I am grateful to H. S. Bear for his interest in this work. He pointed out to me that my original proof of Theorem 1 was valid only for metrizable $K$, and supplied the simple geometric proof of Lemma 4. I am also grateful to the referee for indicating several places where a few more details would substantially improve the exposition.

(2) A stronger version of Lemma 1 follows immediately from Bauer's open mapping theorem (to appear in Equationes Mathematicae, see [3, Theorems 5-13]). 
(3) There remains the problem for general parts: Find a condition (geometric or topological) on a part $\Delta$ of $K$ equivalent to (1).

\section{REFERENCES}

1. Heinz Bauer and H. S. Bear, The part metric in convex sets, Pacific J. Math. 30 (1969), 15-33.

2. H. S. Bear, Continuous selection of representing measures, Bull. Amer. Math. Soc. 76 (1970), 366-369. MR 41 \#2395.

3. - Lectures on Gleason parts, Lecture Notes in Math., vol. 121, SpringerVerlag, Berlin and New York, 1970. MR 41 \#5950.

4. N. V. Har'kova, Generalized Poisson formula, Vestnik Moskov. Univ. Ser. I Mat. Meth. 22 (1967), no. 4, 25-30. (Russian) MR 35 \#4733.

5. J. L. Kelley and I. Namioka, Linear topological spaces, University Series in Higher Math., Van Nostrand, Princeton, N.J., 1963. MR 29 \#3851.

6. R. R. Phelps, Lectures on Choquet's theorem, Van Nostrand, Princeton, N.J., 1966. MR 33 \#1690.

Department of Mathematics, Queen's University, Kingston, Ontario, Canada 